\title{
Multidimensional, multistage wavelet footprints: a new tool for image segmentation and feature extraction in medical ultrasound.
}

\author{
Christian H. P. Jansen ${ }^{1}$, Muthuvel Arigovindan ${ }^{2}$, Michael Sühling ${ }^{2}$, Stefan Marsch ${ }^{1}$, Michael Unser ${ }^{2}$ \\ and Patrick Hunziker ${ }^{1}$ \\ ${ }^{1}$ University Hospital Basel, Switzerland \\ ${ }^{2}$ Swiss Federal Institute of Technology, Lausanne, Switzerland (EPFL)
}

\begin{abstract}
We present a new wavelet-based strategy for autonomous feature extraction and segmentation of cardiac structures in dynamic ultrasound images. Image sequences subjected to a multidimensional (2D plus time) wavelet transform yield a large number of individual subbands, each coding for partial structural and motion information of the ultrasound sequence. We exploited this fact to create an analysis strategy for autonomous analysis of cardiac ultrasound that builds on shape- and motion specific wavelet subband filters. Subband selection was in an automatic manner based on subband statistics. Such a collection of predefined subbands corresponds to the so-called footprint of the target structure and can be used as a multidimensional multiscale filter to detect and localize the target structure in the original ultrasound sequence. Autonomous, unequivocal localization by the autonomous algorithm is then done using a peak finding algorithm, allowing to compare the findings with a reference standard. Image segmentation is then possible using standard region growing operations. To test the feasibility of this multiscale footprint algorithm, we tried to localize, enhance and segment the mitral valve autonomously in 182 non-selected clinical cardiac ultrasound sequences. Correct autonomous localization by the algorithm was feasible in 165 of 182 reconstructed ultrasound sequences, using the experienced echocardiographer as reference. This corresponds to a $91 \%$ accuracy of the proposed method in unselected clinical data. Thus, multidimensional multiscale wavelet footprints allow successful autonomous detection and segmentation of the mitral valve with good accuracy in dynamic cardiac ultrasound sequences which are otherwise difficult to analyse due to their high noise level.
\end{abstract}

Keywords: image segmentation and feature extraction, image reconstruction, cardiac ultrasound, wavelet analysis

\section{INTRODUCTION}

Ultrasound is one of the most widely used imaging modalities in medicine because it is noninvasive, not associated with radiation exposure or potentially toxic contrast agents; in addition ultrasound machines are relatively inexpensive.

In opposite to computer tomography or magnetic resonance imaging, where image data are often "nicer" to work with, ultrasound image sequences pose a major challenge for image segmentation and feature extraction, because they are characterized by a high speckle noise content, large intensity changes within objects, and often discontinuous contours. Further analysis is complicated by the fact that objects and noise may have similar scales, making simple noise filtering approaches problematic ${ }^{1-3}$. Thus, ultrasound imaging has largely remained a field where subjective, at most semiquantitative description is much more important than objective and quantitative analysis, resulting in a high dependence on observer expertise and intraobserver variability.

The development of improved algorithms for successful image segmentation and feature detection in dynamic ultrasound images is an important step in the long-term goal of automated objective and quantitative analysis of dynamic ultrasound images. While cardiac ultrasound is already a key instrument to diagnose and monitor the most frequent lethal disease of the western world, i.e. heart disease, advanced image processing algorithms may even render this exam more suited for earlier, more objective diagnoses by reducing subjectivity of the exam.

In this paper we present a new wavelet-based strategy for autonomous feature extraction and segmentation of cardiac structure in dynamic ultrasound images. In a first experimental setting we chose the mitral valve (the inlet valve of the left heart ventricle) as target for detection by this technique. Experimentally, we tested the reliability of the autonomous detection and segmentation of the mitral valve in 182 nonselected clinical cardiac ultrasound sequences. 


\section{METHODOLOGY}

Recently research in applied mathematics and signal processing has led to the development of powerful wavelet methods for multiscale representation and analysis of signals. In important difference of these new tools compared to traditional Fourier techniques is an improved capability for local analysis because the wavelet basis functions have local support. Applied to medical ultrasound sequences the use of wavelets ${ }^{4}$ allows to separate image content into a large number of individual subbands, each coding for specific structural information (Figure 1a) and motion characteristics (Figure 1b) in regard to scale ${ }^{5}$.

\section{Image reconstruction from individual subbands}

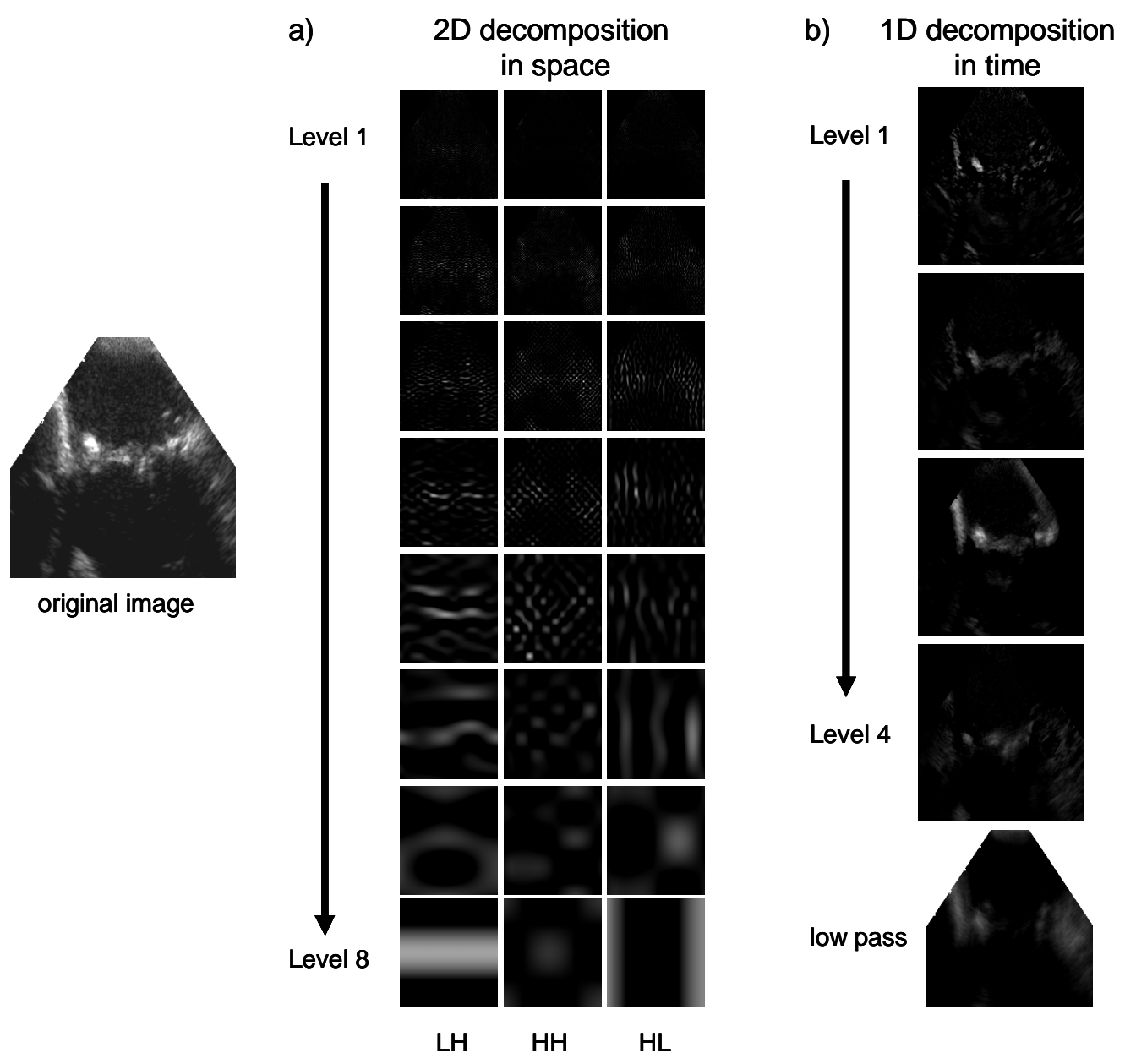

Figure 1. Example of an ultrasound image (left side) wavelet decomposition and reconstruction of individual subbands. a) 2D wavelet transform in space and individual reconstruction of subbands shows structural image features retained in those subbands. b) 1D wavelet transform and reconstruction in time of the corresponding ultrasound sequence of the original image shows motion ultrasound sequence features retained in individual subbands. Note that this is a simplified description of our algorithm design, which combines decomposition in space and time. 
Wavelet packet decomposition ${ }^{6}$, a modification of standard wavelet decomposition, was used in a multiscale approach. Whereas conventional wavelet analysis decomposes only the lowpass subbands iteratively, wavelet packets transform also high pass subbands (Figure 2). Compared to a typical 2D wavelet image decomposition, this leads to a nearly fourfold increase in the number of subbands. Therefore packet wavelet transform yields a huge library of individual subbands giving a high degree of freedom to select subbands that are shape- and motion-specific for the target structure. Because the dimension of the datasets in space and time axis is very different in ultrasound images, they were subjected to different decomposition depths of the wavelet transform, what can easily be done due the separability of the algorithm in individual dimensions. Special care was given to the handling of borders in wavelet decomposition; we used mirror symmetry.
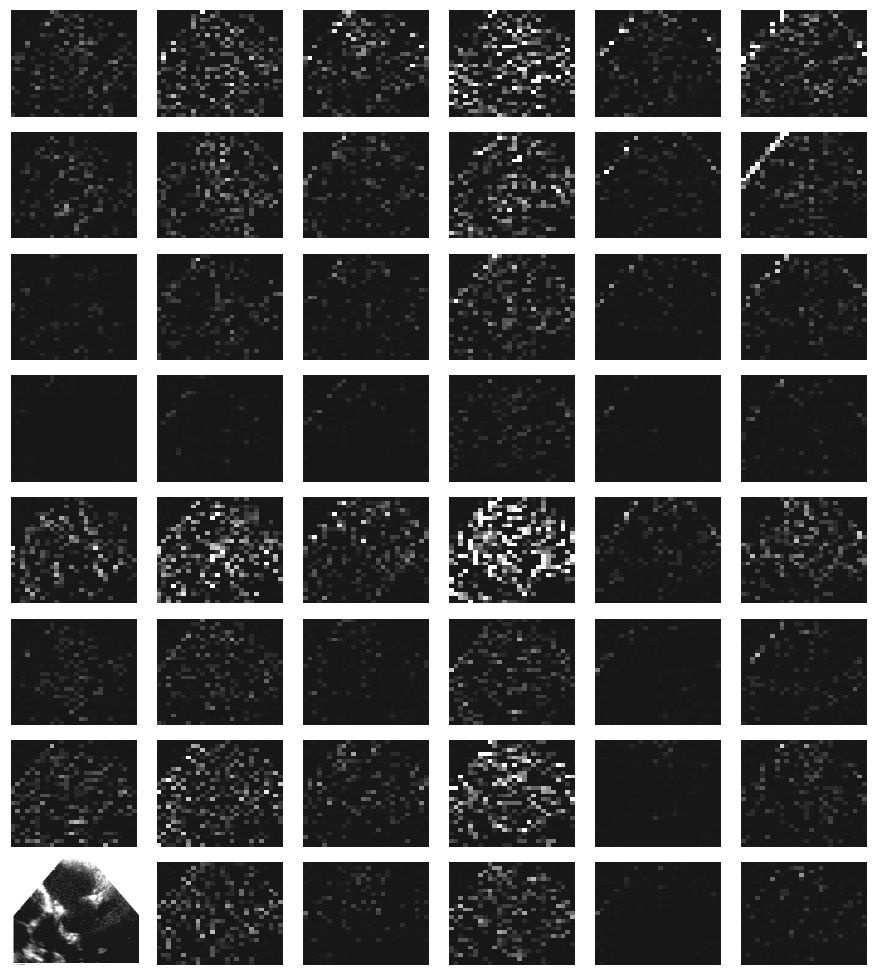
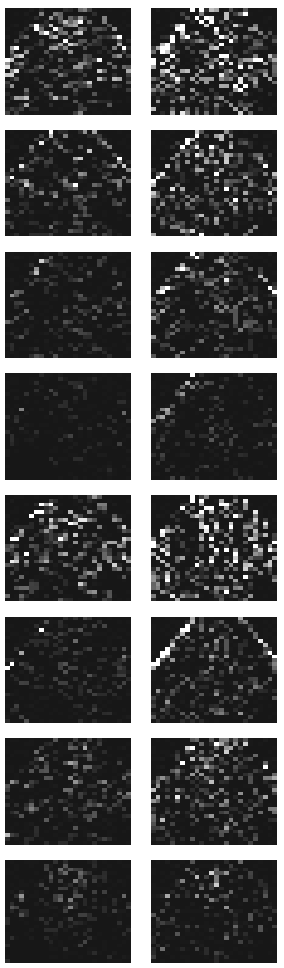

Figure 2. 2D packet wavelet decomposition and image reconstruction from individual subbands of a single ultrasound image. Note that only a three level 2D packet wavelet decomposition is shown, whereas seven decomposition steps in space and four decomposition steps in time were performed consecutively in our algorithm. 


\subsection{Wavelet "footprints"}

Moving cardiac structures have a specific motion pattern and a defined shape. When subjected to a multidimensional (2D plus time) wavelet transform, this yields highly specific "footprints" in the wavelet domain. Based on this observation we created an environment for autonomous analysis of cardiac ultrasound.

To test the feasibility of our algorithm we chose the mitral valve as target structure. The mitral valve as the inlet valve of the left main cardiac chamber is a moving structure. Rapid motion occurs two to three times within the heart cycle corresponding to time scale of about 50 to $200 \mathrm{~ms}$. Valve thickness is about two to three millimeters and lateral dimensions are in centimeter range. The orientation of the valve was perpendicular to the ultrasound beam.

\subsection{Filter construction}

In the first experimental setting we tried to identify the characteristic wavelet footprint of the target structure to the filter bank so that we could build a universal multidimensional multiscale filter to detect and segement the mitral valve autonomously. Therefore we analyzed four independent echo sequences.

Image size in space was 256 times 240 pixel ( 8 bit) and there were on average 25 frames for a complete heart cyle. Seven wavelet packet decomposition levels were performed in space, while in the time dimension, 4 decomposition levels was performed. The subbands of the library were then reconstructed separately for individual examination of their contribution to the target structure. For all reconstructed subbands the sinus-to-noise ratio and energy in comparison to the region of interest of the target structure were calculated.

In order to build the target-specific "footprint" filter, subband selection was done in an automatic manner based on pixel statistics. Those subbands which preserved most of the energy of the target structure with an acceptable SNR were chosen by thresholding. Due to the orthogonality of the used wavelet bases selected subbands could be combined arbitrarily.

\subsection{Autonomous Analysis}

In the following experimental setting we used the newly created footprint filter in 182 non-selected clinical cardiac ultrasound sequences to test the applicability and reliability of our algorithm. Unequivocal localization of the target structure by the autonomous algorithm was performed by using a peak finding algorithm, allowing comparison of the findings with a reference standard. Image segmentation was then performed using standard region growing operations. As gold standard for the correct location of the mitral valve in the cardiac ultrasound sequences valve localization was performed by an experienced echocardiographer.

In addition the "footprint" was also used for shape-specific image enhancement that is useful for further visual analysis. 


\section{RESULTS}

The use of target specific wavelet footprints led to significant enhancement of the predefined structure in every reconstructed ultrasound sequence and significantly suppressed noise and non related cardiac structures as shown in pixel statistics of a mitral valve region of interest versus non-related image regions ( $\mathrm{p}<0.05$ by $\mathrm{t}-\mathrm{Test})$.

Correct autonomous localization by the algorithm was achieved in 165 of 182 reconstructed ultrasound sequences, using the experienced echocardiographer as reference. This corresponds to a $91 \%$ accuracy of the proposed method. At the same time, the algorithm inherently led to marked, selective image enhancement specific for predefined structures as shown in the figure 3. This, in turn, permits reliable automatic image segmentation in this high-noise imaging modality.

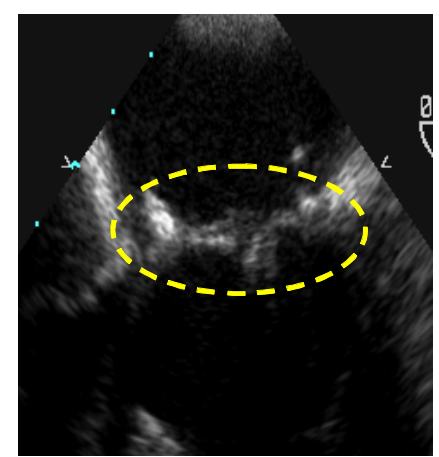

original frame

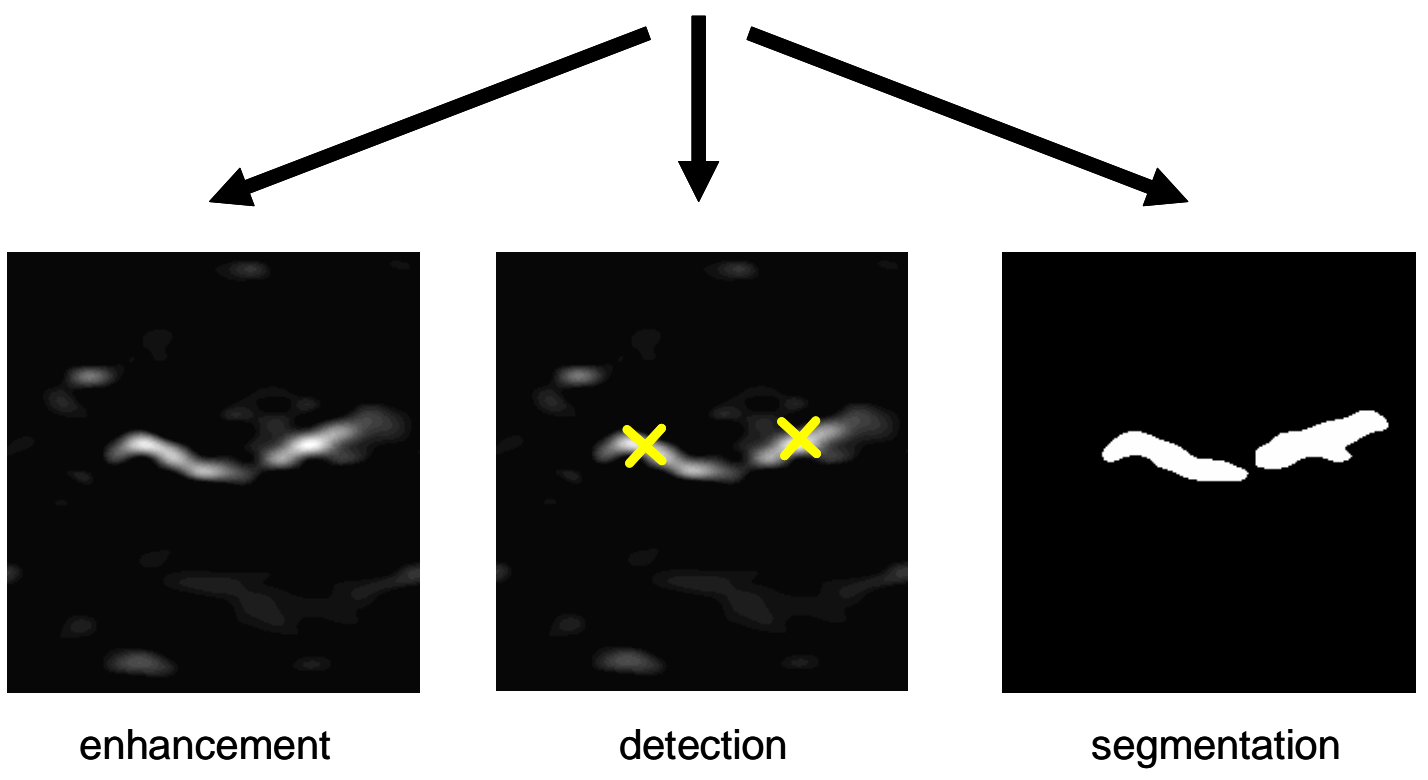

Figure 3. Original frame of dynamic ultrasound sequence (top) during early systole. Use of the multididimensional multiscale wavelet footprint algorithm led to enhancement of the mitral valve and suppression of non related cardiac structures (lower left) and localisation (cross marks) of the mitral valve by the autonomous algorithm (lower middle). Automatic segmentation of the mitral valve (lower right) corresponds well to the true extent of the valve (top). 


\section{LIMITATIONS}

Although prior knowledge about the structure of interest is not necessarily needed to design multiscale, multidimensional filters, there is an intrinsic variability of dimensions in normal and especially in diseased organs. If the tested sample differ markedly from the data used to construct the filters, this may lead to a failure to detect the structure of interest. However, this proved not to be a major problem in our clinical test dataset.

In the current implementation, the algorithm is still sensitive to rotational misalignment. We are currently about to improve this point by adding rotational image registration to our overall strategy.

Computational requirements for multidimensional wavelet analysis with segmentation and feature extraction in large datasets might be a point of concern. Using current PC hardware, computation times of about 60 seconds observed in our experiments for datasets of a size of $\sim 2 \mathrm{MB}$ appear very reasonable. Because wavelets can be computed in $\mathrm{O}(\mathrm{N})$ operations, increasing size of datasets and using a higher number of dimensions is less a concern compared to alternative strategies as e.g. FFT.

\section{CONCLUSION}

This paper presents an algorithm for autonomous image segmentation and feature detection in moving ultrasound images. Multidimensional multiscale wavelet footprints allow successful autonomous feature detection and segmentation with good accuracy in dynamic cardiac ultrasound sequences which are otherwise difficult to analyse due to their high noise level.

Remarkably, in the large number of image sequences tested, this algorithm was highly successful in detection of a feature of interest (the mitral valve) and was able to autonomously localize this object in a large proportion of all tested image despite the known limitations of ultrasound imaging.

User independent objective and quantitative analysis of dynamic ultrasound images is an important advance in dynamic cardiovascular ultrasound, a field where subjective, at most semiquantitative description is still the dominant analysis strategy.

Future extensions of the present work include application to a broader range of target objects looked for in ultrasound images, both in medicine and in other fields. The method appears equally applicable in other imaging modalities where it may complement conventional methods.

\section{REFERENCES}

1. Adam D, Hareuveni O, Sideman S. Semiautomated Border Tracking of Cine Echocardiographic Ventricular Images. IEEE Transactions on Medical Imaging. 1987;6:266-271.

2. Chu CH, Delp EJ, Buda AJ. Detecting Left-Ventricular Endocardial and Epicardial Boundaries by Digital TwoDimensional Echocardiography. IEEE Transactions on Medical Imaging. 1988;7:81-90.

3. Linker DT, Pearlman AS, Lewellen TK, Huntsman LH, Moritz WE. Automated endocardial definition of 2-D echocardiograms: A comparison of four standard edge detectors and improved thresholding techniques. 1, 395-398. 1982. Proc Comput Cardiol.

4. Strang G, Nguyen T. Wavelets and Filter Banks. Wellesley: Camebridge Press, 1997.

5. Mallat S, Hwang WL. Singularity Detection and Processing with Wavelets. IEEE Transactions on Information Theory. 1992;38:617-643.

6. Coifman RR, Wickerhauser MV. Entropy-based algorithms for best basis selection. IEEE on Transactions on Information Theory. 1992;38(2):713-718. 